\title{
Breath Test or Duodenal Aspirate for Small Intestinal Bacterial Overgrowth: Still No Breath of Fresh Air
}

\author{
Eric D. Shah ${ }^{1}$ \\ Published online: 20 August 2020 \\ (c) Springer Science+Business Media, LLC, part of Springer Nature 2020
}

Small intestinal bacterial overgrowth (SIBO) was originally defined by a pathologic increase in bacterial colonization (as determined by culture methods) in the small intestine, which was historically considered a 'sterile' organ [1]. The concept of SIBO originated from studies on patients with postsurgical Billroth II anatomy resulting from stasis of bacteria within the postsurgical blind loop. More recently, SIBO has been associated with symptoms and pathophysiology of irritable bowel syndrome (IBS) [2]. Targeting IBS with rifaximin (or with other antibiotics as an off-label indication) improves global IBS symptoms, based on the presence of SIBO as one of several mechanisms which underlie this complex disorder [3, 4].

Toward developing a better understanding of the strengths and limitations of available SIBO tests, Cangemi et al. in this issue of Digestive Diseases and Sciences retrospectively analyzed 9 years of pooled diagnostic test findings from a quaternary referral center (Mayo Clinic, Jacksonville, Florida) among patients who underwent the two most common diagnostic evaluations for SIBO: breath testing, and duodenal aspirates obtained during a routine upper endoscopy [5]. The primary finding of their study was a significant discrepancy between duodenal aspirate and breath testing results (with disagreement in $37.6 \%$ of cases, $\kappa$ statistic $=-0.02$ ). Considering technical limitations of SIBO testing, the authors conclude that breath testing is more likely the preferred routine option to evaluate for SIBO in clinical practice as a noninvasive, low-cost test.

Breath testing is an ambulatory procedure performed either in-office or at-home through one of several commercial laboratories. The concept of breath testing is based on

Eric D. Shah

eric.d.shah@hitchcock.org

1 Center for Gastrointestinal Motility, Esophageal, and Swallowing Disorders, Section of Gastroenterology and Hepatology, Dartmouth-Hitchcock Medical Center, One Medical Center Drive, Lebanon, NH 03756, USA the exhalation of gases produced solely by the intestinal flora (and not elsewhere in the human body) following ingestion of a carbohydrate substrate. Based on breath testing, SIBO is defined by the rise in $\geq 20$ parts-per-million of hydrogen compared to baseline within 90 min of substrate ingestion as a surrogate for orocecal transit time [6]. Although diagnostic cutoffs improve the practical ease of test interpretation in broader practice, the diagnostic accuracy of breath testing may be limited by differences between breath test findings and orocecal transit confirmed using concurrent scintigraphy, which may affect the interpretable utility of a single cutoff in certain patient populations [7]. Clinicians must also consider differences in test findings based on the two recommended substrates: glucose is less sensitive and more specific, possibly due to its rapid, proximal small intestinal absorption due to active transport which could possibly miss more distal SIBO, compared with lactulose which transits through the small bowel to the colon owing to its resistance to mammalian hydrolases [3, 8-10].

In comparison with breath testing, duodenal aspiration appeals to patients due to its similarity with direct cultures used to confirm common infectious disease diagnoses, and to clinicians due to its original status as the 'gold standard' test for a SIBO diagnosis in Billroth II patients [1]. Unfortunately, duodenal aspirate samples are frequently contaminated, representing $20 \%$ of samples in the current study, which can be explained by the lack of dedicated commercial devices designed to collect aspirates, challenges in avoiding collection of oral flora during tracheoesophageal intubation, and the lack of randomized controlled trials or international consensus protocols addressing appropriate sampling protocols [5].

Interestingly, fewer patients had a positive duodenal aspirate in the current study (16.5\%, compared with $25.9 \%$ of patients by lactulose breath test) [5]. Understanding that the true prevalence of SIBO remains unknown, it is possible that the low frequency of duodenal aspirate positivity may be the result of missed SIBO in the mid- or distal-small bowel. The 
concept of obtaining duodenal aspirates (as opposed to the jejunal or ileal aspirates, or specific sections thereof) relates to the most feasible distal extent of the typical routine upper endoscopy, not necessarily to specific characteristics of the duodenal flora within the broader context of SIBO [2].

Recognizing the detailed characterization of patients in this study, there are two important limitations to consider which relate to the current limited understanding of SIBO itself and changes in definitions of SIBO over time. Diagnostic accuracy studies usually require comparison between a test of interest compared with an index or reference 'gold standard' test, which is not possible when a gold standard for SIBO remains lacking [2]. Furthermore, as with other findings accrued in the growing literature addressing the structure and function of the gut microbiome, the bacterial count may be less important than changes in relative abundance and frequency of specific bacterial phyla, changes that may prove important in human health but the significance of which remains poorly understood [11, 12]. Finally, the authors used a nonstandard definition for a positive duodenal aspirate which required a higher bacterial count $\left(>10^{5}\right.$ colony forming units $/ \mathrm{mL}$ ) compared with the more recent North American Consensus ( $>10^{3}$ colony forming units/ $\mathrm{mL}$ ) [6]. With this lower threshold advocated by the North American Consensus, one could hypothesize that lactulose breath testing may actually be more congruent with the North American Consensus definition-again recognizing that the true prevalence of SIBO remains unknown.

Overall, the lack of clear and precise definitions of SIBO and the lack of a gold standard by which to truly assess diagnostic accuracy of any SIBO test, remain the most important obstacles to the progress of ongoing research efforts. The current findings by Cangemi et al. are important as they add to the literature in support for updated clinical guidance advocating care in making a SIBO diagnosis, recognizing the limitations of existing diagnostic tools [2, 13]. Compared with the duodenal aspirate, breath testing provides a noninvasive, inexpensive means to offer an indirect, surrogate test, recognizing all of inherent limitations of current knowledge. Future, rigorous work is needed to improve the comprehensive understanding of the small intestinal microbiome and its contribution to human health.

Author contributions All authors were involved in drafting the manuscript and approved the final copy.

Funding Eric Shah is supported by the AGA Research Foundation's 2019 AGA-Shire Research Scholar Award in Functional GI and Motility Disorders.

\section{Compliance with Ethical Standards}

Conflict of interest The authors declare that there is no conflict of interests.

\section{References}

1. King CE, Toskes PP. Small intestine bacterial overgrowth. Gastroenterology. 1979;76:1035-1055.

2. Pimentel M, Saad RJ, Long MD, Rao SSC. ACG clinical guideline: small intestinal bacterial overgrowth. Am J Gastroenterol. 2020;115:165-178.

3. Shah A, Talley NJ, Jones M, et al. Small intestinal bacterial overgrowth in irritable bowel syndrome: a systematic review and meta-analysis of case-control studies. Am J Gastroenterol. 2020;115:190-201.

4. Shah ED, Basseri RJ, Chong K, Pimentel M. Abnormal breath testing in IBS: a meta-analysis. Dig Dis Sci. 2010;55:2441-2449.

5. Cangemi DJ, Lacy BE, Wise J. Diagnosing small intestinal bacterial overgrowth: a comparison of lactulose breath tests to small bowel aspirates. Dig Dis Sci. (Epub ahead of print). https://doi. org/10.1007/s10620-020-06484-z.

6. Rezaie A, Buresi M, Lembo A, et al. Hydrogen and methanebased breath testing in gastrointestinal disorders: the North American consensus. Am J Gastroenterol. 2017;112:775-784.

7. Lin EC, Massey BT. Scintigraphy demonstrates high rate of falsepositive results from glucose breath tests for small bowel bacterial overgrowth. Clin Gastroenterol Hepatol. 2016;14:203-208.

8. Wright EM, Loo DDF, Hirayama BA. Biology of human sodium glucose transporters. Physiol Rev. 2011;91:733-794.

9. Saunders, Wiggins HS. Conservation of mannitol, lactulose, and raffinose by the human colon. Am J Physiol-Gastrointest Liver Physiol. 1981;241:397-402.

10. Carulli N, Salvioli GF, Manenti F. Absorption of lactulose in man. Digestion. 1972;6:139-145.

11. Leite GGS, Weitsman S, Parodi G, et al. Mapping the segmental microbiomes in the human small bowel in comparison with stool: a REIMAGINE study. Dig Dis Sci. 2020;65:2595-2604. https:// doi.org/10.1007/s10620-020-06173-x.

12. Saffouri GB, Shields-Cutler RR, Chen J, et al. Small intestinal microbial dysbiosis underlies symptoms associated with functional gastrointestinal disorders. Nat Commun. 2019. https://doi. org/10.1038/s41467-019-09964-7.

13. Quigley EMM, Murray JA, Pimentel M. AGA clinical practice update on small intestinal bacterial overgrowth: expert review. Gastroenterology. 2020. https://doi.org/10.1053/j.gastr o.2020.06.090.

Publisher's Note Springer Nature remains neutral with regard to jurisdictional claims in published maps and institutional affiliations. 